\title{
STEUER
}

\section{Nachhaltigkeit rechnet sich}

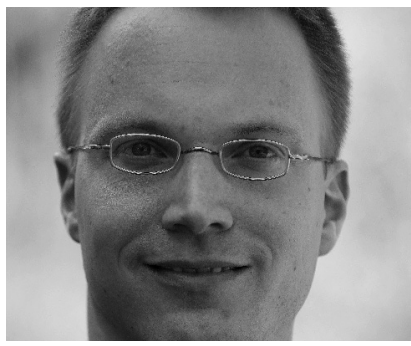

VON MICHAEL LÜHN

Prof. Dr. Michael Lühn studierte Betriebswirtschaftslehre an der Westfälischen Wilhelms-Universität Münster. Nach der Promotion war er Referatsleiter im Rechnungswesen eines großen Energieversorgungsunternehmens. Seit 2009 ist er Inhaber der Professur für Betriebswirtschaftslehre, insbesondere Rechnungswesen und Controlling an der Nordakademie - Hochschule der Wirtschaft - in Elmshorn. Seine Forschungsschwerpunkte sind externe Rechnungslegung und betriebswirtschaftliche Steuerlehre.

E-Mail

michael.luehn@nordakademie.de

\author{
Vor dem Einsatz moderne Energieanlagen müssen ne- \\ ben technischen Fragen auch weitere Aspekte bedacht \\ werden, wie der nachfolgende Beitrag zur steuerrechtli- \\ chen Beurteilung des Betriebs eines Blockheizkraft- \\ werks durch eine gemeinnützige Körperschaft zeigt.
}

Nachhaltigkeit ist zu einem zentralen Aspekt wirtschaftlichen Handelns geworden. Mit einem entsprechenden Konzept können auch gemeinnützige Körperschaften sowohl ihre Effizienz steigern als auch ihr Image verbessern. Ein Element eines Nachhaltigkeitskonzepts kann die nachhaltige Gewinnung von Energie darstellen. Dies kann zum einen durch den Einsatz von regenerativen Energien und zum anderen durch die Erhöhung der Effizienz des Einsatzes fossiler Brennstoffe geschehen.

Einen Beitrag hierzu kann der Einsatz eines Blockheizkraftwerkes (BHKW) darstellen, welches mittels Verfeuerung von fossilen Energieträgern oder Biomasse in einem Verbrennungsmotor Strom und Wärme zur Verfügung stellt. Während in fossilen Großkraftwerken, in denen normalerweise die entstandene Wärme nicht sinnvoll genutzt wird, oftmals nur ein Wirkungsgrad von ca. 50 Prozent erzielt wird, kann bei der Strom- und Wärmeproduktion in einem Blockheizkraftwerk ein Wirkungsgrad von 90 Prozent erreicht werden. Der Einsatz fossiler Energieträger und damit der CO2-Ausstoß können somit erheblich reduziert werden. Der Staat unterstützt den umweltschonenden Betrieb von Blockheizkraftwerk u. a. mittels Zulagen nach dem Kraft-Wärme-Kopplungsgesetz (KWKG).

Die Strom- und Wärmeproduktion durch ein Blockheizkraftwerk kann für gemeinnützige Körperschaften auch steuerliche Konsequenzen nach sich ziehen. Insbesondere sind die Fragen zu klären, ob der Betrieb des Blockheiz- kraftwerks einen wirtschaftlichen Geschäftsbetrieb darstellt und ob der Betreiber ein Unternehmer im Sinne des Umsatzsteuergesetzes ist. Diesen Fragen wird im Folgenden nachgegangen.

\section{Wirtschaftlicher Einsatz eines Blockheizkraftwerk}

Der Einsatz von Blockheizkraftwerk kann zu einer deutlichen Reduzierung der Energiegewinnungskosten führen. Aufgrund des hohen Wirkungsgrades von 90 Prozent kann insbesondere Strom wesentlich günstiger produziert werden als der Strombezug über einen Energieversorger. So liegen die Stromgestehungskosten bei einem erdgasbefeuerten Blockheizkraftwerk unter Zugrundelegung eines Erdgaspreises von 5 ct./ KWh und sonstigen Betriebskosten von $2 \mathrm{ct} . / \mathrm{KWh}$ bei einem Wert von unter 8 ct./KWh. Darüber hinaus gibt es zahlreiche weitere finanzielle Vorteile des Einsatzes von Blockheizkraftwerk:

- Gewährung eines Zuschlags nach dem Kraft-Wärme-Kopplungsgesetz bis zu 5,11 ct./KWh

- Erstattung der Energiesteuer auf die eingesetzten fossilen Brennstoffe

- Rückerstattung der Stromsteuer auf den eigenverbrauchten Strom und

- Befreiung von der Umlage nach dem Erneuerbare Energien-Gesetz (EEG), die zu Beginn des Jahres 2011 von 2,047 ct./KWh auf 3,530 ct./KWh erhöht wurde. 
Diesen Vorteilen bei den variablen Kosten der Stromgestehung stehen die hohen Investitionskosten gegenüber. Bei einem Blockheizkraftwerk mit einer elektrischen Leistung von $20 \mathrm{KW}$ und einer thermischen Leistung von $45 \mathrm{KW}$ muss beispielweise von einer Investitionssumme inklusive Einbindung der Anlage in das vorhandene Heizungssystem von 60.000 Euro zzgl. Umsatzsteuer gerechnet werden. Um die Amortisationszeit möglichst gering zu halten, sollte das Blockheizkraftwerk so ausgelegt werden, dass es mindestens 5.500 Stunden im Jahr unter Volllast laufen kann. Folglich sollte in mindestens sieben Monaten des Jahres ein Wärmebedarf in Höhe der thermischen Leistung des Blockheizkraftwerk vorhanden sein. Darüber hinaus ist es vorteilhaft, wenn ein Großteil des Stroms selbstverbraucht wird, da dann die hohen Strombezugskosten vom Energieversorger eingespart werden können.

\section{Vergütungen nach dem Kraft- Wärme-Kopplungsgesetz}

Der Betrieb von Blockheizkraftwerk wird durch das Kraft-Wärme-Kopplungsgesetz finanziell gefördert. Die Höhe der finanziellen Förderung richtet sich gem. $\$ 5 \mathrm{KWKG}$ insbesondere nach dem Zeitpunkt der Inbetriebnahme und nach der Leistung der Anlage. Im Folgenden sollen nur kleine, hocheffiziente (1) Kraft-Wärme-Kopplungsanlage (KWK) - Anlagen i. S. des $\$ 3$ Abs. 2, 3 und $11 \mathrm{KWKG}$ betrachtet werden. Als klein im Sinne des Kraft-Wärme-Kopplungsgesetz gilt eine Anlage, wenn sie eine elektrische Leistung von maximal zwei MW aufweist. Wurde eine derartige Anlage ab dem 1. Januar 2009 bis zum 31. Dezember 2016 errichtet, so sieht das Kraft-Wärme-Kopplungsgesetz folgende Förderungen vor:

- Der Netzbetreiber ist gem. $\mathbb{S} 4$ Abs. 1 und 3 KWKG verpflichtet, den Strom von KWK-Anlagen abzunehmen und mindestens mit dem durchschnittlichen Preis für Grundlaststrom an der Strombörse EEX in Leipzig im jeweils vorangegangenen Quartal zu vergüten (zurzeit ca. 3,5 bis $5 \mathrm{ct} . / \mathrm{KWh}$ ).

- Darüber hinaus ist gem. $\mathbb{4}$ Abs. 3 Satz 1 i. V. m. Abs. 3a KWKG für den gesamten produzierten Strom ein $\mathrm{Zu}$ schlag zu entrichten, der vom Netzbe- treiber an den Betreiber der KWKAnlage zu zahlen ist. Die Zuschlagssätze sind gem. $\mathbb{S} 7$ Abs. 5 und 6 KWKG nach Leistungsanteilen gestaffelt: Leistungsanteil bis 50 $\mathrm{kWel}$ : $5,11 \mathrm{ct} . / \mathrm{KWh}$ über einen maximalen Förderzeitraum von zehn Jahren, Leistungsanteil über $50 \mathrm{kWel}$ bis 2 MWel: 2,1 ct./KWh über einen maximalen Förderzeitraum von sechs Jahren und höchstens 30.000 Vollbenutzungsstunden.

Um die Förderungen zu erhalten, ist ein Antrag auf Zulassung der KWK-Anlage gem. $\$ 6$ KWKG beim Bundesamt für Wirtschaft und Ausfuhrkontrolle (BAFA) zu stellen. (2)

\section{Energiesteuerrückerstattung und Befreiung von der Stromsteuer}

Betreiber von KWK-Anlagen können für steuerpflichtige Energieerzeugnisse (Brennstoffe) gem. $\$ 53$ EnergieStG i. V. m. $\$ 98$ EnergieStV einen Antrag beim Hauptzollamt auf Rückerstattung der Energiesteuer einreichen. Sofern der von einem kleinen Blockheizkraftwerk mit einer elektrischen Nennleistung von bis zu zwei Megawatt erzeugte Strom im räumlichen Zusammenhang zu der Anlage verbraucht wird, ist dieser gem. $\mathbb{9}$ Abs. 1 Nr. 3 StromStG von der Stromsteuer i. H. v. 2,05 ct./KWh befreit. Ein räumlicher Zusammenhang wird angenommen, wenn Blockheizkraftwerk und Verbraucher an demselben Transformator angeschlossen sind, so dass der Strom nicht in das regionale Mittelspannungs-Netz eingespeist werden muss.

\section{Ertragsteuerliche Beurteilung}

Gemeinnützige Körperschaften i. S. der $\mathbb{S} 51$ bis $68 \mathrm{AO}$ sind gem. $\$ 5$ Abs. 1 Nr. 9 Satz 1 KStG von der Körperschaftsteuer und gem. $\mathbb{3}$ Nr. 6 Satz 1 GewStG von der Gewerbesteuer befreit.

Fraglich ist, ob der Betrieb des Blockheizkraftwerk einen wirtschaftlichen

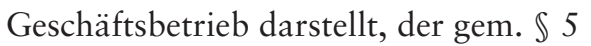
Abs. 1 Nr. 9 Satz 2 KStG bzw. $\$ 3$ Nr. 6 Satz 2 GewStG von der Steuerbefreiung ausgenommen ist. Ein wirtschaftlicher Geschäftsbetrieb liegt nach $\mathbb{1 4} \mathrm{AO}$ bei einer selbständigen, nachhaltigen Tätigkeit, durch die Einnahmen oder andere wirtschaftliche Vorteile erzielt werden und die über den Rahmen einer Vermö- gensverwaltung hinausgeht. Eine Gewinnerzielungsabsicht ist nicht notwendig. Zu analysieren ist hier insbesondere, ob die Einspeisung des überschüssigen Stroms des Blockheizkraftwerk in das allgemeine Stromnetz eine nachhaltige Tätigkeit darstellt. Nach neuerer Rechtsprechung des Bundesfinanzhofes liegt eine nachhaltige Tätigkeit vor, wenn eine auf Dauer angelegte Tätigkeit (im weitesten Sinne gewerbliche oder berufliche Tätigkeit) vorliegt. Für deren Beurteilung ist das Gesamtbild der Verhältnisse maßgebend, bei der die Merkmale, die für oder gegen die Nachhaltigkeit sprechen, abzuwägen sind. (3)

Bei der Einspeisung des überschüssigen Stroms eines Blockheizkraftwerk in das Stromnetz liegt unzweifelhaft eine mehrjährige Tätigkeit, eine auf Wiederholung angelegte Tätigkeit und die Ausführung mehr als nur eines Umsatzes vor, da vor dem Betrieb des Blockheizkraftwerk mit dem Netzbetreiber ein Einspeisevertrag, der auf unbestimmte Zeit zur Lieferung des überschüssigen Stroms verpflichtet, abzuschließen ist. Wird die Anlage so dimensioniert, dass regelmäßig Strom in nennenswerten Mengen in das allgemeine Stromnetz eingespeist wird, so muss von einer nachhaltigen Tätigkeit ausgegangen werden, die einen gewerblichen Geschäftsbetrieb i. S. d. $\mathbb{} 14 \mathrm{AO}$ darstellt. In diesem Fall unterliegen die Einkünfte aus dem Blockheizkraftwerk der Körperschaftsteuer und der Gewerbesteuer, sofern sie die Freigrenze von 35.000 Euro gem. $\$ 55$ Abs. $3 \mathrm{AO}$ übersteigen.

Eine nur gelegentliche, eher zufällig ausgeführte Tätigkeit, die aufgrund der fehlenden Nachhaltigkeit keinen gewerblichen Geschäftsbetrieb begründet, liegt hingegen vor, wenn

- aufgrund der Lastprofile aus der Vergangenheit davon auszugehen ist, dass eine Stromeinspeisung nur bei nicht planmäßigen Betrieb der gemeinnützigen Körperschaft zustande kommt (beispielsweise geringer Stromverbrauch aufgrund des Ausfalls elektrischer Geräte)

- damit im Vorhinein nicht abgeschätzt werden kann, wann Stromüberschüsse entstehen, die ins Netz eingespeist werden und

- das Blockheizkraftwerk ausschließlich wärmegeführt wird. Die Strom- 
produktion ist somit völlig unabhängig von den aktuell zu erzielende bzw. einzusparenden Strompreisen.

Liegen diese Voraussetzungen vor, so sind die Entgelte für den Verkauf des überschüssigen Stroms und die Zahlung des KWK-Zuschlags auf den selbstgenutzten Strom keine Einnahmen aus einem wirtschaftlichen Geschäftsbetrieb. Es handelt sich vielmehr um Zuschüsse auf die Kosten der Energieversorgung, die für die Aufrechterhaltung der gemeinnützigen Tätigkeit notwendig sind und somit nicht der Körperschaftsteuer und Gewerbesteuer unterliegen.

\section{Umsatzsteuerliche Beurteilung}

Selbstgenutzer Strom: Gemeinnützige Körperschaften sind nicht allgemein von der Umsatzsteuer befreit. Häufig üben gemeinnützige Körperschaften jedoch Tätigkeiten aus, die unter eine Steuerbefreiung des $\mathbb{4} 4$ UStG fallen. Die Bereitstellung von Strom und Wärme ist i. d. R. notwendige Voraussetzung zur Erbringung der steuerbefreiten Umsätze. Die Zahlung des Zuschlags gem. \$ 4 Abs. 3 Satz 1 i. V. m. Abs. 3a KWKG auf den selbstgenutzten Strom ist nicht umsatzsteuerbar, da kein Leistungsaus- tausch zwischen BlockheizkraftwerkBetreiber und Netzbetreiber vorliegt. Da gem. \4 Abs. $1 \mathrm{KWKG}$ nur eine Pflicht zur Abnahme des überschüssigen Stroms durch den Netzbetreiber besteht, ist nicht davon auszugehen, dass der Strom zunächst an den Netzbetreiber geliefert und anschließend vom Netzbetreiber wieder zurückgeliefert wird. (4)

Verkauf von Wärme und Strom an Dritte: Wird die vom Blockheizkraftwerk erzeugte Wärme nicht ausschließlich selbst genutzt, sondern teilweise an Dritte vermarktet, so liegt eine nachhaltige gewerbliche Tätigkeit vor, die die Unternehmereigenschaft gem. $\$ 2$ Abs. 1 UStG begründet. Die Veräußerung ist somit gem. $\mathbb{S} 1$ Abs. $1 \mathrm{Nr} .1$ UStG steuerbar. Ob sie auch steuerpflichtig ist, hängt insbesondere von den Vertragsbeziehungen zum Abnehmer ab. Handelt es sich beispielsweise bei dem Abnehmer um einen Mieter oder Pächter von Grundstücken der gemeinnützigen Körperschaft, so stellt die Bereitstellung von Wärme und Warmwasser eine im unmittelbaren Zusammenhang stehende übliche Nebenleistung für die nach $\mathbb{S} 4 \mathrm{Nr} .12 \mathrm{UStG}$ steuerbefreite Vermietungs- oder Verpachtungsleistung dar, die gem. Tz. 4.12.1 Abs. 5 Satz 3 UStAE ebenfalls von der Umsatzsteuer befreit ist. Sofern auch Strom an den jeweiligen Mieter oder Pächter verkauft werden soll, ist dieser ebenfalls als Nebenleistung von der Umsatzsteuer befreit. (5) Wird hingegen die vom Blockheizkraftwerk produzierte Wärme oder der Strom an Dritte veräußert, die nicht Mieter oder Pächter von Grundstücken der gemeinnützigen Körperschaft sind, so sind die Erlöse hieraus mangels Steuerbefreiung der Umsatzsteuer zu unterwerfen.

Verkauf von Strom an den Netzbetreiber: Fraglich ist, wie der Verkauf von überschüssigem Strom an den Netzbetreiber umsatzsteuerlich zu beurteilen ist. Zunächst ist zu prüfen, ob die gemeinnützige Körperschaft als Unternehmer i. S. v. $\$ 2$ UStG tätig wird. Das entscheidende Kriterium ist hier wiederum die Nachhaltigkeit der Tätigkeit zur Erzielung von Einnahmen. Insofern kann auf die Ausführungen zur Beurteilung des Vorliegens eines wirtschaftlichen Geschäftsbetriebs i. S. v. $\$ 14$ AO verwiesen werden. Sofern der Strom regelmäßig gegen Entgelt in das allgemeine Stromnetz eingespeist wird, ist folglich von einer nachhaltigen Tätigkeit auszugehen, die die Unternehmereigenschaft des Betreibers begründet. (6) Im Um-

\section{Wo Blockheizkraftwerke in der Sozialwirtschaft eingesetzt werden können}

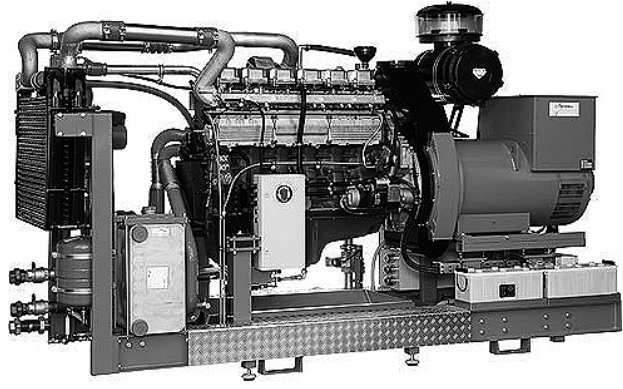

Ein Blockheizkraftwerk (BHKW) ist eine moderne Anlage zur Gewinnung elektrischer Energie und Wärme, die vor allem am Ort des Verbrauchs selbst betrieben werden soll, aber auch zusätzlich Nutzwärme in ein Nahwärmenetz einspeisen kann. Sie setzt dazu das Prinzip der Kraft-Wärme-Kopplung ein. In der Sozialwirtschaft können dafür typischerweise verschiedene Bereich infrage kommen:

- Krankenhäuser: Krankenhäuser weisen einen kontinuierlichen Strombedarf auf, der im Verhältnis zum Wärmebedarf größer ausfällt als bei anderen Einrichtungen. In einem Krankenhaus mit 400 Betten und einem Wärme- und Strom- bedarf von je $5.000 \mathrm{MW}$ im Jahr könnte beispielsweise ein Blockheizkraftwerk mit $100 \mathrm{~kW}$ elektrischer Leistung und 200 kW thermischer Leistung auf eine hohe Zahl von Volllaststunden kommen.

- Bildungseinrichtungen: Der Einsatz von Blockheizkraftwerk ist für Bildungseinrichtungen insbesondere dann sinnvoll, wenn der erzeugte Strom selbst genutzt werden kann. Ein Blockheizkraftwerk sollte jedoch nur einen kleinen Teil des Wärmebedarfs abdecken, um möglichst viele Voll laststunden zu erzielen. Eine Schule mit 200 MWh Strombedarf und 500 MWh Wärmebedarf sollte höchstens mit einem Blockheizkraftwerk mit $20 \mathrm{~kW}$ elektrischer Leistung und $40 \mathrm{~kW}$ thermischer Leistung ausgestattet werden, um einen wirtschaftlichen Einsatz zu gewährleisten.

- Beherbergungseinrichtungen: Da neben der Raumwärme ein Wärmebedarf in der Küche und im Sanitärbereich vorhanden ist, kann das Blockheizkraftwerk auch in den Sommermonaten eingesetzt werden. Für eine Jugendherberge mit einem Wärmebedarf von $600 \mathrm{MWh}$ im Jahr und einem Strombedarf von 200 MWh im Jahr könnte ein Blockheizkraftwerk mit $30 \mathrm{~kW}$ elektrischer Leistung und $60 \mathrm{~kW}$ thermischer Leistung eingesetzt werden. 
kehrschluss sind die Umsätze aus dem Verkauf des eingespeisten Stroms bei nur gelegentlicher Einspeisung des Stroms mangels Unternehmereigenschaft nicht steuerbar im Rahmen der Umsatzsteuer.

Vorsteuerabzug: Gem. $\mathbb{S} 15$ Abs. 2 Satz 1 Nr. 1 UStG ist die Steuer für die Lieferungen von Gegenständen sowie für die sonstigen Leistungen, die der Unternehmer zur Ausführung von steuerfreien Umsätzen verwendet, vom Vorsteuerabzug ausgeschlossen. Sofern das Blockheizkraftwerk fast ausschließlich zur Bereitstellung von Strom und Wärme für die steuerbefreiten Leistungen der gemeinnützigen Körperschaft betrieben wird, ist somit ein Vorsteuerabzug ausgeschlossen. Sofern der Verkauf von überschüssigen Strom an den Netzbetreiber und die Vereinnahmung von $\mathrm{Zu}$ schlägen auf den selbstverbrauchten Strom nicht steuerbar ist (s. o.), kommt auch für diesen Anteil kein Vorsteuerabzug infrage. Wird der Strom und/oder die Wärme hingegen teilweise in einem steuerpflichtigen Vorgang veräußert, so ist für diesen Teil ein Vorsteuerabzug gem. $\mathbb{S} 15$ Abs. 4 UStG möglich. Die Aufteilung in abziehbare und nicht abziehbare Teilbeträge ist sachgerecht durchzuführen. Sachgerecht wäre eine Aufteilung nach den produzierten Energiemengen. Für den Anteil an den produzierten Energiemengen, der steuerpflichtig veräußert wird, ist dann ein Vorsteuerabzug möglich.

\section{Zusammenfassung}

Der Einsatz eines Blockheizkraftwerk für die Strom- und Wärmeerzeugung gemeinnütziger Körperschaften ist insbesondere dann wirtschaftlich sinnvoll, wenn eine hohe Zahl an Volllaststunden erzielt werden kann. Bei einer Wirtschaftlichkeitsanalyse sind jedoch auch die steuerlichen Konsequenzen $\mathrm{zu}$ berücksichtigen. Sofern die in einem Blockheizkraftwerk produzierten Strom- und Wärmemengen für die steuerbefreiten Tätigkeiten einer gemeinnützigen Körperschaft eingesetzt werden, stellt der Betrieb des Blockheizkraftwerk keinen gewerblichen Geschäftsbetrieb i. S. v. $\mathbb{1} 14 \mathrm{AO}$ dar. Wird der produzierte Strom nur gelegentlich in das allgemeine Stromnetz eingespeist, liegt auch keine Unternehmereigenschaft i. S. v. $\mathbb{} 2$ USt $G$ vor. In diesem Fall sind der Betrieb des Blockheizkraftwerk und die gelegentliche Erzielung von Einnahmen aus der Stromeinspeisung im Rahmen der Körperschaftsteuer, der Gewerbesteuer und der Umsatzsteuer steuerbefreit. Ein Vorsteuerabzug ist damit ebenfalls ausgeschlossen.

Wird hingegen nicht nur gelegentlich Strom in das allgemeine Stromnetz eingespeist oder ein Teil des Stroms und/oder der Wärme an Dritte veräußert, liegt für diese Teile der Stromund Wärmeproduktion ein gewerblicher Geschäftsbetrieb vor, der körperschaftsteuer- und gewerbesteuerpflichtig ist. Darüber hinaus wird die Steuerbarkeit im Sinne des UStG begründet. In diesem Fall ist der Teil der Vorsteuer, der auf die nicht steuerbefreiten Umsätze entfällt, abzugsfähig.

\section{Anmerkungen}

(1) Das Merkmal "hocheffizient " richtet sich nach der Richtlinie 2004/8/EG des Europäischen Parlaments und des Rates vom 11. Februar 2004 über die Förderung einer am Nutzwärmebedarfs orientierten KraftWärme-Kopplung im Energiebinnenmarkt und zur Änderung der Richtlinie 92/42/EWG, Abl. EU Nr. L 52, S. 50.

(2) Nähere Erläuterungen zur Zulassung im Internet unter: http://www.bafa.de/bafa/de/energie/kra

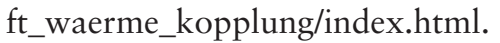

(3) Vgl. erstmals BFH vom 18.07.1991 - V R 86/87, BStBl II 1991, 776; eine Auflistung der Merkmale findet sich in Tz. 2.3 Abs. 5 Satz 4 UStAE.

(4) Von einer Lieferung und Rücklieferung geht hingegen das BMF beim Eigenverbrauch von EEG-geförderten Photovoltaikanlagen aus, vgl. BMF vom 01.04.2009 - IV B 8 - S 7124/07/10002, vgl. hierzu auch Fromm, DStR 2010, 207.

(5) Vgl. BFH-Urteil vom 15.01.2009, V R 91/07, DStRE 2009, 615, BMFSchreiben vom 21.07.2009, IV B 9 - S 7168/08/10001, DStR 2009, 1586 sowie Aufhebung von A 76 Abs. 6 Satz 1 UStr.

(6) Vgl. BFH vom 18.12.2008, V R 80/07, BB 2009, 803; siehe auch Tz. 2.5 Abs. 1 UStAE für die Einspeisung von Strom aus EEG-Anlagen.

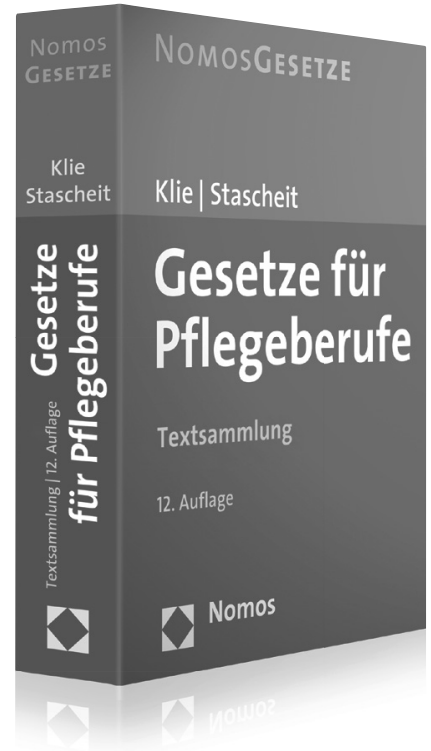

\section{Gesetze für Pflegeberufe}

Textsammlung

Herausgegeben von

Prof. Dr. Thomas Klie und

Prof. Ulrich Stascheit

12. Auflage 2011, 1.235 S.,

brosch., 22,-€

ISBN 978-3-8329-6052-0

Die umfassend aktualisierte Textsammlung, Stand 15. November 2010, wurde in der 12. Auflage u.a. erweitert um

- die Behindertenrechtskonvention

- das FamFG

- die Durchführungsanweisungen mit Hinweisen zu Haushaltshilfen

- eine Synopse zum Landesheimrecht

- das Wohn- und Betreuungsvertragsgesetz

- die Empfehlungen BEK und DHPV

»eine gute Orientierungshilfe»

Dr. Thomas P. Stähler, Deutsche Rentenversicherung 6/o6, zur Vorauflage

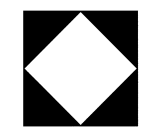

Nomos 